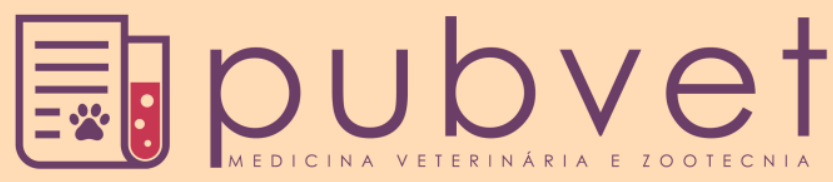

https://doi.org/10.22256/pubvet.v12n6al11.1-6

\title{
Obtenção de oócitos em cadelas e gatas submetidas a ovariosalpingohisterectomia
}

\author{
Artur da Nóbrega Carreiro ${ }^{\bullet} 1 *$, Joyce Galvão de Souza ${ }^{\ominus 2}$, Ana Yasha Ferreira de \\ La Salles ${ }^{\bullet}$, Brunna Muniz Rodrigues Falcão ${ }^{\ominus 2}$, Débora Vitória Fernandes de \\ Araújo ${ }^{3}$, João Augusto Rodrigues Alves Diniz ${ }^{2}$, Ediane Freitas Rocha ${ }^{1}$, \\ Norma Lúcia de Souza Araújo ${ }^{\bullet}$, Danilo José Ayres de Menezes 5
}

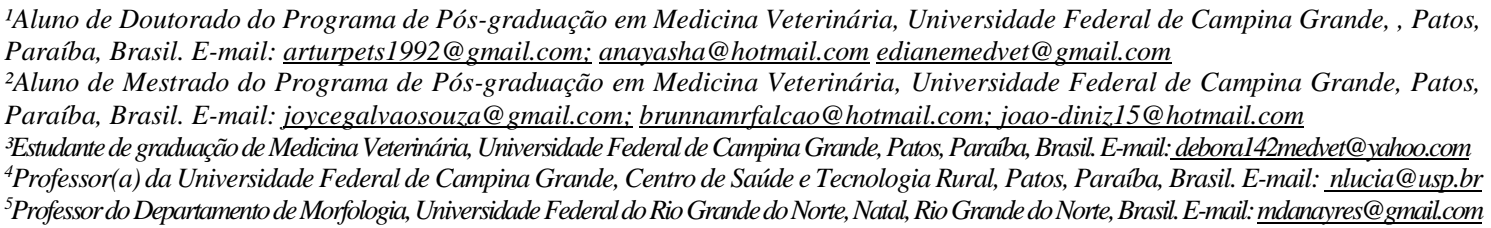

RESUMO. A utilização de técnicas modernas para aplicação na área da reprodução cresce cada vez mais na medicina veterinária e com isso se fazem necessários estudos que avaliem a eficiência destas técnicas. Devido à localização cortical dos folículos dentro do ovário, a técnica mais adequada para obtenção dos oócitos caninos é o Slicing, o qual apresenta melhores resultados em relação a quantidade de oócitos coletados quando comparado com outras técnicas. Buscou-se com este estudo, verificar a qualidade dos oócitos coletados em ovários de cadelas e gatas com diferentes idades que foram submetidas à procedimento de Ovário Salpingo Histerectomia (OSH) no Hospital Veterinário da Universidade Federal de Campina Grande. Os oócitos foram classificados quanto a sua qualidade com relação a morfologia do complexo cumulus oophorus. A idade e a fase estral em que as cadelas e gatas se encontravam no momento da coleta não influenciou na qualidade e quantidade dos oócitos coletados.

Palavras chave: cadelas, gatas, reprodução, slicing

\section{Obtaining oocytes in puppies and cats submitted to salpingo- oophorectomy}

\begin{abstract}
The use of modern techniques for application in the field of reproduction grows more and more in veterinary medicine and therefore studies are needed to evaluate the efficiency of these techniques. Due to the cortical location of the follicles within the ovary, Slicing is the most adequate technique to obtain canine oocytes, which presents better results in relation to the amount of oocytes collected when compared to other techniques. The aim of this study was to verify the quality of oocytes collected from ovaries of bitches and cats with different ages submitted to the Salpingo Ovary Hysterectomy (OSH) procedure at the Veterinary Hospital of the Federal University of Campina Grande. The oocytes were classified according to their quality in relation to the morphology of the cumulus oophorus complex. The age and estrus stage in which bitches and cats were at the time of collection did not influence the quality and quantity of oocytes collected.
\end{abstract}

Keywords: puppies, cats, reproduction, slicing 


\section{Obtención de ovocitos en perras y gatas sometidas a ovario salpingo histerectomía}

RESUMEN. La utilización de técnicas modernas para aplicación en el área de la reproducción crece cada vez más en la medicina veterinaria y con ello se hacen necesarios estudios que evalúen la eficiencia de estas técnicas. Debido a la localización cortical de los folículos dentro del ovario, la técnica más adecuada para la obtención de los ovocitos caninos es el Slicing, el cual presenta mejores resultados en relación a la cantidad de ovocitos recogidos en comparación con otras técnicas. Se buscó con este estudio verificar la calidad de los ovocitos recogidos en ovarios de perras y gatas con diferentes edades que fueron sometidas al procedimiento de Ovario Salpingo Histerectomía (OSH) en el Hospital Veterinario de la Universidade Federal de Campina Grande. Los ovocitos se clasificaron en cuanto a su calidad con relación a la morfología del complejo cumulus oophorus. La edad y la fase estral en que las perras y gatas se encontraban en el momento de la recolección no influenció en la calidad y cantidad de los ovocitos recolectados.

Palabras clave: perras, gatos, reproducción, rebanar

\section{Introdução}

A Biotecnologia da reprodução tem trazido inúmeras contribuições à Medicina Veterinária (Albuquerque et al. 2012). O manejo reprodutivo assistido que tem sido uma grande ferramenta para o desenvolvimento e aprimoramento do conhecimento a respeito da fisiologia da reprodução. No manejo reprodutivo assistido, podemos destacar a fecundação in vitro, a qual proporciona uma análise mais detalhada dos mecanismos fisiológicos reprodutivos, da capacidade reprodutiva, além da possibilidade de intervir com técnicas e substâncias a serem testadas (Prestes 2005; Rubin et al. 2009).

Para que se faça a fecundação in vitro é de fundamental importância o emprego de técnicas eficientes de coleta de gametas, como a coleta de oócitos, a qual pode ser feita pela punção, digestão ovariana e o fatiamento ovariano; este último, conhecido como Slicing. Todas estas técnicas têm por objetivo único obter o maior número de oócitos íntegros e aptos para a fertilização in vitro. Em canídeos, os folículos ficam localizados na região cortical do tecido ovariano, tornando-se aparentes poucos dias antes do momento da ovulação. Isto dificulta o uso da técnica de aspiração folicular, método rotineiramente aplicado em outras espécies (Pires 2006). Assim, a técnica empregada para a obtenção de oócitos caninos é o Slicing ou fatiamento, que permite a liberação de maior número de oócitos quando comparada à aspiração folicular (Nickson et al. 1993).
Neste contexto, constituem-se como objetivos, realizar um estudo da qualidade dos oócitos nas diferentes faixas etárias em cadelas e gatas submetidas a Ovário Salpingo Histerectomia (OSH) no Hospital Veterinário do Centro de Saúde e Tecnologia Rural da Universidade Federal de Campina Grande verificando possíveis diferenças na qualidade dos oócitos coletados pela técnica Slicing nas duas espécies submetidas a Ovário Salpingo Histerectomia (OSH) no Hospital Veterinário do Centro de Saúde e Tecnologia Rural da Universidade Federal de Campina Grande.

\section{Material e Métodos}

O experimento foi realizado no Laboratório de Reprodução Animal do Hospital Veterinário (HV) e no Laboratório de Microscopia do Centro de Saúde e Tecnologia Rural (CSTR) da Universidade Federal de Campina Grande (UFCG). Foram utilizadas 12 cadelas e 12 gatas submetidas a ovariosalpingoesterectomia (OSH) no Centro Cirúrgico do Hospital Veterinário do Centro de Saúde Tecnologia Rural da UFCG. Previamente à cirurgia, foram coletados os dados do histórico clinico do animal em uma ficha. Em seguida, realizou-se o exame de citologia vaginal através do esfregaço com um swab estéril em lâmina microscópica corada pelo Panótico rápido para análise e identificação da fase do ciclo estral do animal. Após a cirurgia, o ovário esquerdo e direito foram dissecados e conduzidos em tubos do tipo Falcon de $15 \mathrm{~mL}$ contendo solução fisiológica de Cloreto de Sódio a $0,9 \%$ e imediatamente conduzidos em caixa de 
isopor ao Laboratório de Reprodução Animal e ao laboratório de Microscopia da UAMV. No laboratório, os ovários foram pesados em balança de precisão da marca AAKERBS 3000 A Bivolt - Bioprecisa ${ }^{\circledR}$. E em seguida medidos quanto à largura e comprimento com o auxílio de um paquímetro. Após isso, mantidos em placas de Petri com solução fisiológica de Cloreto de Sódio a 0,9\%, para serem "fatiados" com lâminas de bisturi $\mathrm{n}^{\circ} 24$ em cortes com 1 a $2 \mathrm{~mm}$ de espessura, com auxílio de uma lupa estereomicroscópica para melhor visualização dos oócitos. Em seguida, os oócitos foram coletados com o auxílio de uma micropipeta e repassados para outra placa com solução fisiológica de cloreto de sódio a $0,9 \%$ e em seguida, avaliados quanto à sua qualidade. O critério avaliativo utilizado foi a qualidade dos oócitos coletados com relação a morfologia do Complexo cumulusoóforos (COCS), sendo classificados em Grau 1(G1) com pigmentação escura com uma ou mais camadas de células do cumulus, Grau 2 (G2) com pigmentação clara com camadas incompletas de células do cumulus, Grau 3 (G3) com pigmentação pálida, sem formato definido e sem células do cumulus aderidas, além da quantidade de oócitos coletados por ovário (Hewitt \& England 1997).

\section{Resultados e Discussão}

De acordo com a tabela 1 pôde-se observar que, das 12 cadelas utilizadas neste estudo, seis apresentavam piometra. Isso ocorreu devido ao fato de os animais serem trazidos ao HV apenas quando apresentam histórico de enfermidade.

Ta bela 1. Qualidade dos oócitos coletados em cadelas submetidas a Ováriosalpingohisterectomia no HV/CSTR/UFCG com suas respectivas características clínicas. DP: Desvio Padrão; S/A: Sem alterações; SRD: Sem Raça Definida; AC: Uso de anticoncepcional; O.D.: Ovário direito; O.E.: Ovário esquerdo; G1: Oócitos de grau 1; G2: Oócitos de grau 2; G3: Oócitos de grau 3.

\begin{tabular}{|c|c|c|c|c|c|c|c|c|c|c|c|c|}
\hline Animal & $\begin{array}{c}\text { Peso, } \\
\text { kg }\end{array}$ & $\begin{array}{l}\text { Idade } \\
\text { (anos) }\end{array}$ & Raça & $\begin{array}{c}\text { Histórico } \\
\text { Clínico }\end{array}$ & Partos & $\mathrm{AC}$ & $\begin{array}{c}\text { Fase do } \\
\text { ciclo }\end{array}$ & Ovário & G1 & $\mathrm{G} 2$ & G3 & Total \\
\hline \multirow{2}{*}{01} & \multirow{2}{*}{28,0} & \multirow{2}{*}{3} & \multirow{2}{*}{ Rotweiller } & \multirow{2}{*}{ S/A } & \multirow{2}{*}{0} & \multirow{2}{*}{ Não } & \multirow{2}{*}{ Anestro } & O.E. & 0 & 4 & 10 & 14 \\
\hline & & & & & & & & O.D. & 1 & 2 & 9 & 12 \\
\hline \multirow{2}{*}{02} & \multirow{2}{*}{9,6} & \multirow{2}{*}{4} & \multirow{2}{*}{ Pinscher } & \multirow{2}{*}{ Piometra } & \multirow{2}{*}{0} & \multirow{2}{*}{ Sim } & \multirow{2}{*}{ Diestro } & O.E. & 21 & 37 & 43 & 101 \\
\hline & & & & & & & & O.D.. & 25 & 34 & 53 & 102 \\
\hline \multirow{2}{*}{03} & \multirow{2}{*}{9,0} & \multirow{2}{*}{4} & \multirow{2}{*}{ Poodle } & \multirow{2}{*}{ Distocia } & \multirow{2}{*}{1} & \multirow{2}{*}{ Não } & \multirow{2}{*}{ Diestro } & O.E. & 6 & 8 & 7 & 21 \\
\hline & & & & & & & & O.D. & 6 & 9 & 4 & 19 \\
\hline \multirow{2}{*}{04} & \multirow{2}{*}{5,75} & \multirow{2}{*}{10} & \multirow{2}{*}{ Poodle } & \multirow{2}{*}{ Piometra } & 0 & $\operatorname{Sim}$ & Diestro & O.E. & 7 & 3 & 19 & 29 \\
\hline & & & & & & SIIII & DIEstro & O.D. & 5 & 4 & 20 & 29 \\
\hline 05 & 305 & 2 & SPD & Distopis & 1 & & & O.E. & 18 & 14 & 12 & 44 \\
\hline 05 & 3,95 & 2 & SRD & Distocia & 1 & Não & Diestro & O.D. & 6 & 4 & 6 & 16 \\
\hline 06 & & & CDP & & 0 & & & O.E. & 37 & 9 & 29 & 75 \\
\hline 06 & 16,6 & 7,8 & SRD & P1ometra & 0 & Nao & Anestro & O.D. & 40 & 15 & 13 & 68 \\
\hline 07 & 100 & 2 & SPD & Piometro & 0 & $\operatorname{Sim}$ & Diectro & O.E. & 18 & 34 & 46 & 98 \\
\hline 07 & 10,0 & 2 & SRD & Piometra & 0 & Sim & Diestro & O.D.. & 35 & 17 & 60 & 112 \\
\hline 08 & 67 & 6 & SRD & Hiperplasia & 1 & Não & Anestro & O.E. & 25 & 12 & 51 & 88 \\
\hline 08 & 0,1 & 0 & SKD & Mamária & 1 & Nao & Anestro & O.D. & 15 & 21 & 44 & 80 \\
\hline 00 & 140 & 8 & Poodle & Piometro & 1 & $\operatorname{Sim}$ & Diectro & O.E. & 3 & 7 & 37 & 47 \\
\hline 09 & 14,0 & 0 & Foodie & Fiometra & 1 & SIIII & DIEstro & O.D. & 10 & 4 & 25 & 39 \\
\hline 10 & 115 & 11 & SRD & Distocia & 1 & Não & Diestro & O.E. & 7 & 2 & 7 & 16 \\
\hline 10 & $11, J$ & 1,1 & SKD & Distocia & 1 & Nao & DIEstro & O.D. & 4 & 1 & 10 & 15 \\
\hline 11 & 60 & 4 & Pinscher & Piometra & 0 & $\operatorname{Sim}$ & Diestro & O.E. & 10 & 22 & 44 & 76 \\
\hline & & & & & & & & O.D. & 19 & 13 & 52 & 84 \\
\hline 12 & 111 & 7 & SPD & Piometro & 0 & & & O.E. & 7 & 19 & 32 & 38 \\
\hline 12 & 11,1 & 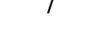 & SRD & Piometra & 0 & Sim & Diestro & O.D. & 4 & 10 & 40 & 54 \\
\hline Média & 11,02 & 4,91 & & & & & & & 13,71 & 12,71 & 28,04 & 53,21 \\
\hline DP & $\pm 6,45$ & $\pm 2,81$ & & & & & & & $\pm 11,67$ & $\pm 10,57$ & $\pm 18,23$ & $\pm 33,37$ \\
\hline
\end{tabular}


$\mathrm{Na}$ sua grande maioria, os casos de piometra estão associados ao uso de anticoncepcionais de forma indiscriminada. Quanto aos oócitos coletados, ainda de acordo com a tabela 1 , obteve-se uma média de $28 \pm 18,2$ oócitos de Grau 3 (G3), refletindo mais que o dobro em relação as médias de oócitos de Grau 1 (G1) $(13,7 \pm 11,6)$ e Grau $2(\mathrm{G} 2)(12,7 \pm 10,5)$. A alta variabilidade de oócitos coletados entre as cadelas pôde ser constatada pelo valor do desvio padrão (DP) obtido para esse parâmetro.

No tocante à fase do ciclo estral, a maioria das cadelas encontrava-se nas fases de anestro e diestro. Nos casos onde houveram distocias, neste trabalho, a fase do ciclo estral foi classificada como diestro gestacional e, nas demais, por apresentarem quadro de piometra, sendo uma enfermidade específica da fase de diestro.

A quantidade de gatas sem alterações clínicas (S/A), conforme os dados contidos na tabela 2, foi maior quando comparado com a quantidade de cadelas saudáveis demonstrada na tabela 1. Esse resultado pode ser devido ao fato de os proprietários das gatas estarem mais atentos quanto à necessidade de se realizar a OSH eletiva para evitar a prenhez indesejada nessa espécie. A média da quantidade total de oócitos Grau 3 (G3) $(21,1 \pm 13,5)$ nas gatas foi superior a quantidade de oócitos Grau 1 (G1) $(11,4 \pm 7,9)$ e Grau 2 (G2) (10,2 $\pm 6,3)$, semelhantemente ao que ocorreu nas cadelas. Os valores de desvio padrão obtidos neste estudo revelam uma grande variação na quantidade de oócitos coletados, estando de acordo com Lopes et al. (2004) onde, em um trabalho sobre a avaliação da maturação nuclear in vitro de oócitos de gatas domésticas pré-púberes e púberes constatou uma variação muito alta do número de oócitos coletados(de três a 72 oócitos) entre as fêmeas.

Tabela 2. Qualidade dos oócitos coletados em gatas submetidas à Ováriosalpingohisterectomia no HV/CSTR/UFCG com suas respectivas características clínicas. DP: Desvio Padrão; S/A: Sem alterações; SRD: Sem Raça Definida; AC: Uso de anticoncepcional; O.D.: Ovário direito; O.E.: Ovário esquerdo; G1: Oócitos de grau 1; G2: Oócitos de grau 2; G3: Oócitos de grau 3.

\begin{tabular}{|c|c|c|c|c|c|c|c|c|c|c|c|c|}
\hline$\overline{\text { Animal }}$ & $\begin{array}{l}\text { Peso } \\
(\mathrm{Kg})\end{array}$ & $\begin{array}{l}\text { Idade } \\
\text { (anos) }\end{array}$ & Raça & $\begin{array}{c}\text { Histórico } \\
\text { clínico }\end{array}$ & Partos & $\mathrm{AC}$ & $\begin{array}{c}\text { Fase do } \\
\text { ciclo }\end{array}$ & Ovário & G1 & $\mathrm{G} 2$ & G3 & Total \\
\hline \multirow[t]{2}{*}{01} & 3,0 & 1,6 & SRD & S/A & 0 & Não & Anestro & O.E. & 12 & 17 & 28 & 57 \\
\hline & & & & & & & & O.D. & 7 & 14 & 14 & 35 \\
\hline \multirow[t]{2}{*}{02} & 2,8 & 2 & SRD & S/A & 0 & Não & Diestro & O.E. & 16 & 9 & 29 & 54 \\
\hline & & & & & & & & O.D. & 6 & 13 & 27 & 46 \\
\hline \multirow[t]{2}{*}{03} & 2,6 & 2 & SRD & S/A & 1 & Não & Diestro & O.E. & 7 & 10 & 14 & 31 \\
\hline & & & & & & & & O.D. & 4 & 7 & 13 & 24 \\
\hline \multirow[t]{2}{*}{04} & 3,1 & 18 & SRD & S/A & 1 & Não & Diestro & O.E. & 0 & 3 & 6 & 9 \\
\hline & & & & & & & & O.D. & 7 & 5 & 9 & 21 \\
\hline \multirow[t]{2}{*}{05} & 2,8 & 0,7 & SRD & S/A & 0 & Não & Anestro & O.E. & 10 & 12 & 16 & 38 \\
\hline & & & & & & & & O.D. & 5 & 10 & 26 & 41 \\
\hline \multirow[t]{2}{*}{06} & 3,5 & 3 & SRD & S/A & 0 & Sim & Anestro & O.E. & 8 & 7 & 10 & 25 \\
\hline & & & & & & & & O.D. & 11 & 9 & 14 & 34 \\
\hline \multirow[t]{2}{*}{07} & 2,1 & 0,9 & SRD & Piometra & 0 & Não & Diestro & O.E. & 13 & 18 & 39 & 70 \\
\hline & & & & & & & & O.D. & 2 & 2 & 7 & 11 \\
\hline \multirow[t]{2}{*}{08} & 4,25 & 4 & SRD & S/A & 3 & Sim & Diestro & O.E. & 26 & 25 & 53 & 104 \\
\hline & & & & & & & & O.D. & 36 & 25 & 60 & 121 \\
\hline \multirow[t]{2}{*}{09} & 2,6 & 2 & SRD & Distocia & 1 & Sim & Diestro & O.E. & 13 & 6 & 14 & 33 \\
\hline & & & & & & & & O.D. & 15 & 4 & 20 & 39 \\
\hline \multirow[t]{2}{*}{10} & 5,2 & 1,5 & SRD & S/A & 0 & Sim & Anestro & O.E. & 12 & 6 & 25 & 33 \\
\hline & & & & & & & & O.D. & 17 & 8 & 17 & 42 \\
\hline \multirow[t]{2}{*}{11} & 3,8 & 4 & SRD & S/A & 1 & Sim & Anestro & O.E. & 22 & 15 & 18 & 55 \\
\hline & & & & & & & & O.D. & 8 & 12 & 22 & 42 \\
\hline \multirow[t]{2}{*}{12} & 5,0 & 3 & SRD & Piometra & 0 & Sim & Diestro & O.E. & 6 & 8 & 17 & 31 \\
\hline & & & & & & & & O.D. & 11 & 1 & 10 & 22 \\
\hline Média & 3,40 & 3,41 & & & & & & & 11,42 & 10,25 & 21,17 & 42,42 \\
\hline DP & $\pm 0,98$ & $\pm 2,35$ & & & & & & & $\pm 7,96$ & $\pm 6,37$ & $\pm 13,5$ & $\pm 5,93$ \\
\hline
\end{tabular}


Pode-se constatar que, numericamente, houve um discreto aumento no número de oócitos de grau 1 coletados nas cadelas com idade superior a cinco anos neste experimento, em relação ao mesmo tipo de oócito em cadelas com idade inferior a cinco anos. Por sua vez, houve um maior número de oócitos de grau 3 (G3) em cadelas com menos de cinco anos de idade.

Ao comparar os oócitos G1 e G3, observase uma maior quantidade de oócitos de Grau 3 (G3) nas cadelas em quase todas as faixas etárias avaliadas neste estudo. Songsasen \&Wildt (2007) afirmaram que a melhor idade para a obtenção de oócitos de boa qualidade cadelas é entre 1 e 6 anos e que fêmeas de raça pura apresentam significativamente um menor número de oócitos viáveis do que fêmeas cruzadas entre raças diferentes. Observa-se que houve em termos numéricos, uma maior quantidade dos oócitos $\mathrm{G} 2$ coletados nas gatas com idade a partir de 2 anos em relação as gatas com idade inferior a 2 anos. Lopes et al. (2004) em seu estudo sobre a qualidade morfológica do oócito concluíram que as gatas com idade entre um e três anos são doadoras mais aptas para coleta de oócitos de boa qualidade. No presente trabalho, as gatas não apresentaram diferença na quantidade de oócitos Grau 1 (G1), sendo que os oócitos tipo $3 \mathrm{G}(3)$, apresentaram-se em maior quantidade que os G1 nas duas faixas etárias avaliadas neste estudo, resultado semelhante ao encontrado nas cadelas. Ainda de acordo com os resultados relativos à quantidade de oócitos G1, G2 e G3 coletados em cadelas e em gatas, os mesmos foram numericamente semelhantes entre os ovários esquerdo e direito nas duas espécies.

No presente trabalho obtivemos uma média de 52,14 oócitos para cadelas abaixo de 5 anos e 54,71 oócitos para cadelas acima de 5 anos. Esses números diferem dos obtidos por Tucholski et al. (2008) que, avaliaram a quantidade de oócitos obtida em fêmeas caninas de diferentes faixas etárias em diestro e anestro, com um grupo composto de cadelas de 1 a 2,5 anos de idade (jovens) e outro grupo composto por cadelas com 3 a 8 anos de idade (adultas) e obteve um total de 70 oócitos, com média de 12,6 para o grupo de cadelas jovens e um total de 68 oócitos para o grupo de cadelas adultas. Esse resultado, provavelmente ocorreu pela forma como as faixas etárias foram divididas no presente trabalho.

\section{Conclusão}

De acordo com os resultados obtidos e segundo as condições deste estudo, pode-se concluir que: observou-se variação numérica entre a quantidade de oócitos de grau 1 (G1), grau 2 (G2) e grau 3 (G3) de oócitos obtidos nas diferentes faixas etárias das cadelas e gatas avaliadas neste estudo; aparentemente, a fase do ciclo estral não influenciou na qualidade e quantidade de oócitos coletados nas duas espécies estudadas; o estado de saúde das fêmeas avaliadas neste estudo, aparentemente, não influenciou a qualidade e a quantidade de oócitos coletados. Não houve diferença entre a quantidade e a qualidade de oócitos coletados entre os ovários direito e esquerdo das cadelas e gatas utilizadas neste trabalho. Mais estudos são necessários para avaliar a influência de outros fatores na qualidade de oócitos coletados de cadelas e gatas.

\section{Referências Bibliográficas}

Albuquerque K.P., Prado I.N., Prado R.M., Cavallieri F.L.B., Rigolon L.P. \& Barbosa O.R. 2012. Superovulatory response, production and quality of embryos of cows fed on linseed or canola seed supplemented diets. Acta Scienciarum. Animal Sciences 34, 321-7.

Hewitt D.A. \& England G.C. 1997. Effect of preovulatory endocrine events upon maturation of oocytes of domestic bitches. Journal of Reproduction and Fertility Supplement 51, 83-91.

Lopes M.D., Uiechi E. \& Trinca L.A. 2004. Avaliação da maturação nuclear in vitro de oócitos de gatas domésticas (Felis catus) pré-púberes e púberes. Brazilian Journal of Veterinary Research and Animal Science 41, 268-73.

Nickson D.A., Boyd J.S., Eckersall P.D., Ferguson J.M., Harvey M.J. \& Renton J.P. 1993. Molecular biological methods for monitoring oocyte maturation and in vitro fertilization in bitches. Journal of Reproduction and Fertility Supplement 47, 231-40.

Pires A. 2006. Efeito da suplementação de cisteína e cisteamina sobre a maturação nuclear de oócitos de fêmeas caninas (Canis familiaris) obtidos por ovariosalpingohisterectomia durante a fase pré-ovulatória do estro. Universidade Estadual Paulista 
Júlio de Mesquita Filho, Jaboticabal, São Paulo.

Prestes N.C. 2005. Produção in vitro de embriões bovinos-problemas e desafiosVisão obstétrica. Acta Scientiae Veterinariae 33, 119-24.

Rubin M.I.B., Pessoa G.A., Fraga D.R., F.F. V. \& Silva C.A.M. 2009. Produção in vitro de embriões e clonagem: um caminho conhecido? Revista brasileira de reprodução animal 6, 77-85.

Songsasen N. \& Wildt D.E. 2007. Oocyte biology and challenges in developing in vitro maturation systems in the domestic dog. Animal reproduction science 98, 2-22.
Tucholski Â.P., Raschelli D.L., Nogueira Morais R., Angeli A.L., Rocha T.M.M. \& Tanaka N. 2008. Quantidade de oócitos obtidos em fêmeas caninas de diferentes faixas etárias em diestro e anestro. Revista Acadêmica: Ciência Animal 6, 341-7.

\author{
Article History: \\ Received 4 April 2018 \\ Accepted 26 April 2018 \\ Available online 31 May 2018
}

License information: This is an open-access article distributed under the terms of the Creative Commons Attribution License 4.0, which permits unrestricted use, distribution, and reproduction in any medium, provided the original work is properly cited. 